\title{
On the commutativity conditions for rings and T-rings
}

\author{
Hatice Kandamar (D), Okan Arslan* ${ }^{*}$ \\ Department of Mathematics, Adnan Menderes University, Aydın, Turkey
}

\begin{abstract}
Let $R$ be any ring. In this paper we observe the relation between the center of $R$-ring $R$ and the center of usual ring $R$ and then prove if the center of $R$-ring $R$ is nonzero, then $R$ is commutative as a ring. We also show that the common hypothesis

$$
a \alpha b \beta c=a \beta b \alpha c \text { for all } a, b, c \in M \text { and } \alpha, \beta \in \Gamma
$$

for a weak Nobusawa $\Gamma$-ring $M$ is sufficent for $M$ to be commutative. Also, we investigate some conditions on ideals of $\Gamma$-ring that make $M$ to be commutative.
\end{abstract}

Mathematics Subject Classification (2010). 16N60, 16U80, 16 Y99

Keywords. commutativity, prime ring, prime $\Gamma$-ring, ideal

\section{Introduction and preliminaries}

The notion of a gamma ring introduced by Nobusawa as a ternary algebraic system in 1964. After the definition of a gamma ring that has been given by Barnes in 1966 by weakened Nobusawa's definition, many algebraists focused on the study of gamma rings.

Prime and semiprime ideals for gamma rings was firstly introduced by Barnes [1] in 1966 and by Kyuno [5] in 1975 respectively. They proved the main structural properties of primeness and semiprimeness.

Commutativity conditions for usual rings are one of the common topic that the mathematicians work and there have been obtained a number of theorems to determine the conditions for a prime ring (or semiprime ring) to be commutative. Analogous to usual rings, commutativity conditions for $\Gamma$-rings are important and there have been many results concerning conditions that force a $\Gamma$-ring to be commutative. When we look at some of the papers investigating commutativity conditions for $\Gamma$-rings we come across the below assumption:

$$
a \alpha b \beta c=a \beta b \alpha c \text { for all } a, b, c \in M \text { and } \alpha, \beta \in \Gamma
$$

There are gamma rings that satisfy the assumption $(*)$. For example, let $M$ is the set of all $2 \times 3$ matrices of the form $\left[\begin{array}{lll}a & 0 & b \\ 0 & c & 0\end{array}\right]$ and $\Gamma$ is the set of all $3 \times 2$ matrices of the form

\footnotetext{
* Corresponding Author.

Email addresses: hkandamar@adu.edu.tr (H. Kandamar), oarslan@adu.edu.tr (O. Arslan)

Received: 08.10.2017; Accepted: 08.12.2019
} 
$\left[\begin{array}{ll}0 & 0 \\ 0 & y \\ 0 & 0\end{array}\right]$ for $a, b, c, y \in \mathbb{Z}$. Then, it is easy to show that $M$ is a prime Nobusawa $\Gamma$-ring which satisfies $(*)$.

Let $M$ be a $\Gamma$-ring in the sense of Barnes, $a, b \in M$ and $\alpha \in \Gamma$. The commutator of $a$ and $b$ with respect to $\alpha$ is equal to $a \alpha b-b \alpha a$ and denoted by $[a, b]_{\alpha}$. The basic commutator identity for a $\Gamma$-ring $M$ is

$$
[a \alpha b, c]_{\beta}=a \alpha[b, c]_{\beta}+[a, c]_{\beta} \alpha b+a \alpha c \beta b-a \beta c \alpha b
$$

for $a, b, c \in M$ and $\alpha, \beta \in \Gamma$. With the assumption $(*)$, this identity reduces to

$$
[a \alpha b, c]_{\beta}=a \alpha[b, c]_{\beta}+[a, c]_{\beta} \alpha b
$$

for $a, b, c \in M$ and $\alpha, \beta \in \Gamma$. Under the assumption $(*)$, the basic commutator identity for a $\Gamma$-ring $M$ is almost same as the commutator identity for usual rings. Therefore, by performing minor modifications to any proof for a usual ring, one can easily get results for gamma rings. This is the main reason for assuming $(*)$.

The assumption $(*)$ was first seen in the papers [3] and [4] which have many results about the center of a gamma ring. However, it is so strong that without any other assumption it makes any prime weak Nobusawa $\Gamma$-ring $M$ commutative which means the center of $M$ is equal to the $M$. Therefore, investigating commutativity conditions under the assumption $(*)$ is not necessary for prime weak Nobusawa $\Gamma$-rings. Moreover, obtaining $M$ to be a Nobusawa $\Gamma^{\prime}$-ring from a Barnes $\Gamma$-ring $M$ and primeness of $M$ being hereditary tell us that one have to be careful when investigating commutativity conditions in prime Barnes $\Gamma$-rings with the assumption $(*)$.

The main interest of this paper is to investigate commutativity conditions for gamma rings and to observe relation between commutativity of a gamma ring and commutativity of a usual ring. We show that the center of a prime $\Gamma$-ring $M$ in the sense of Nobusawa is either zero or $M$. Then, observing the relation between the center of $R$-ring $R$ and the center of the ring $R$, we prove if the center of $R$-ring $R$ is nonzero, then $R$ is commutative as a ring. In the last part, we investigate some conditions on ideals of $\Gamma$-ring that make $M$ commutative.

Now we give some definitions and basic facts about gamma rings.

Let $M$ and $\Gamma$ be additive Abelian groups. $M$ is said to be a $\Gamma$-ring in the sense of Barnes [1] and denoted by $(\Gamma, M)_{B}$ if there exists a mapping $M \times \Gamma \times M \rightarrow M$ satisfying these two conditions for all $a, b, c \in M, \alpha, \beta \in \Gamma$ :

(1) $(a+b) \alpha c=a \alpha c+b \alpha c$ $a(\alpha+\beta) c=a \alpha c+a \beta c$ $a \alpha(b+c)=a \alpha b+a \alpha c$

(2) $(a \alpha b) \beta c=a \alpha(b \beta c)$

In addition, if there exists a mapping $\Gamma \times M \times \Gamma \rightarrow \Gamma$ such that the following axioms hold for all $a, b, c \in M, \alpha, \beta \in \Gamma$ :

(3) $(a \alpha b) \beta c=a(\alpha b \beta) c$

(4) $a \alpha b=0$ for all $a, b \in M$ implies $\alpha=0$ where $\alpha \in \Gamma$

then $M$ is called a $\Gamma$-ring in the sense of Nobusawa [7] and denoted by $(\Gamma, M)_{N}$. If a $\Gamma$-ring $M$ in the sense of Barnes satisfies only the condition (3), then it is called weak Nobusawa $\Gamma$-ring [6] and denoted by $(\Gamma, M)_{w N}$.

Let $M$ be a $\Gamma$-ring in the sense of Barnes. A subgroup $U$ of $M$ is called left ideal(resp. right ideal) if $M \Gamma U \subseteq U$ (resp. $U \Gamma M \subseteq U$ ). If $U$ is both left and right ideal, the $U$ is called an ideal of $M$. An ideal $\Omega$ of $M$-ring $\Gamma$ is defined similarly. Recall that an ideal $P$ of $M$ is called prime ideal if $a \Gamma M \Gamma b \subseteq P$ for $a, b \in M$ implies $a \in P$ or $b \in P$. An ideal $Q$ of $M$ is called semiprime ideal if $a \Gamma M \Gamma a \subseteq Q$ for $a \in M$ implies $a \in Q$. $M$ is said to 
be a prime (resp. semiprime) $\Gamma$-ring if the zero ideal of $M$ is prime (resp. semiprime). An ideal $S$ of $M$ is called completely prime ideal if $a \Gamma b \subseteq S$ for $a, b \in M$ implies $a \in S$ or $b \in S$. $M$ is said to be completely prime $\Gamma$-ring if the zero ideal of $M$ is completely prime. Clearly, every completely prime $\Gamma$-ring is prime. For a $\Gamma$-ring $M$ in the sense of Nobusawa, the primeness and completely primeness are equivalent.

Let $M$ be a $\Gamma$-ring in the sense of Barnes, $A$ be any subset of $M$ and $\Omega$ be any subset of $\Gamma$. Then, the set $C_{\Omega}(A)=\{x \in M \mid x \beta a=a \beta x, \forall a \in A, \beta \in \Omega\}$ is called the $\Omega$-center of $A$ and the set $C_{A}(\Omega)=\{\gamma \in \Gamma \mid \gamma a \beta=\beta a \gamma, \forall a \in A, \beta \in \Omega\}$ is called the $A$-center of $\Omega$. These subsets are subgroups of $M$ and $\Gamma$ respectively.

Lemma 1.1 ([6, 1.2.1]). If $M$ is a $\Gamma$-ring in the sense of Nobusawa, then $\Gamma$ is a weak Nobusawa M-ring.

Lemma $1.2([6,1.2 .2])$. If $M$ is a weak Nobusawa $\Gamma$-ring, then $M$ is a $\Gamma / \Lambda$-ring in the sense of Nobusawa where $\Lambda=\{\gamma \in \Gamma: M \gamma M=0\}$.

Lemma 1.3 ([2]). Every Barnes $\Gamma$-ring $M$ is a $\Gamma^{\prime}$-ring in the sense of Nobusawa for some Abelian group $\Gamma^{\prime}$.

Proof. Let $\Omega$ is the free Abelian group generated by $\Gamma \times M \times \Gamma$ and $\Lambda$ is the subgroup consisting of all elements $\sum_{i} n_{i}\left(x \alpha_{i} a_{i}\right) \beta_{i} y=0$ for every $x, y \in M$. Now, define $\Gamma^{\prime}$ as the quotient group $\Omega / \Lambda$ and write $[\alpha, a, \beta]$ for the coset $(\alpha, a, \beta)+\Lambda$. Therefore, one can obtain $M$ to be $\Gamma^{\prime}$-ring in the sense of Nobusawa. Here, the ternary multiplications are defined by

$$
x\left(\sum_{i}\left[\alpha_{i}, a_{i}, \beta_{i}\right]\right) y=\sum_{i}\left(x \alpha_{i} a_{i}\right) \beta_{i} y
$$

and

$$
\left(\sum_{i}\left[\alpha_{i}, a_{i}, \beta_{i}\right]\right) x\left(\sum_{i}\left[\gamma_{j}, b_{j}, \delta_{j}\right]\right)=\sum_{i, j}\left[\alpha_{i},\left(a_{i} \beta_{i} x\right) \gamma_{j} b_{j}, \delta_{j}\right]
$$

for all $\sum_{i}\left[\alpha_{i}, a_{i}, \beta_{i}\right]$ and $\sum_{i}\left[\gamma_{j}, b_{j}, \delta_{j}\right]$ in $\Gamma^{\prime}$ and $x, y \in M$.

Remark 1.4. According to the proof of Lemma 1.3, we have $A \Gamma^{\prime} B=A \Gamma M \Gamma B$ for any subsets $A$ and $B$ of $M$. Hence, it is clear that primeness is hereditary under the transition of $(\Gamma, M)_{B}$ to $\left(\Gamma^{\prime}, M\right)_{N}$.

Lemma 1.5. $M$ is a prime $\Gamma$-ring in the sense of Nobusawa if and only if $\Gamma$ is a prime Nobusawa M-ring.

Proof. We only treat the case $M$ is a prime $\Gamma$-ring in the sense of Nobusawa, the other case can be treated similarly.

In the light of Lemma 1.1, it suffices to show that the $M$-ring $\Gamma$ is prime and $\alpha x \beta=0$ for all $\alpha, \beta \in \Gamma$ implies $x=0$. First, assume that $\alpha x \beta=0$ for all $\alpha, \beta \in \Gamma$. Hence, $a \alpha x \beta b=0$ for all $a, b \in M$ and $\alpha, \beta \in \Gamma$. Hence, we get $x=0$ since $M$ is a prime $\Gamma$-ring. Now let $\alpha M \beta=0$ for $\alpha, \beta \in \Gamma$. Therefore,

$$
\begin{aligned}
\alpha M \beta=0 & \Rightarrow(M \alpha M) \Gamma(M \beta M)=0 \\
& \Rightarrow M \alpha M=0 \vee M \beta M=0 \\
& \Rightarrow \alpha=0 \vee \beta=0
\end{aligned}
$$

since $M$ is a prime Nobusawa $\Gamma$-ring. Consequently, $\Gamma$ is a prime $M$-ring in the sense of Nobusawa. 


\section{Commutativity of prime gamma rings and rings}

Lemma 2.1. Let $M$ be a prime Nobusawa $\Gamma$-ring. If $a \alpha b \beta c=a \beta b \alpha c$ for all $a, b, c \in M$ and $\alpha, \beta \in \Gamma$, then $(\Gamma, M)_{N}$ is commutative.

Proof. Since $M$ is a prime $\Gamma$-ring in the sense of Nobusawa, $\Gamma$ is a prime Nobusawa $M$-ring by Lemma 1.5 and

$$
\begin{aligned}
a \alpha b \beta c=a \beta b \alpha c & \Rightarrow a(\alpha b \beta-\beta b \alpha) c=0 \\
& \Rightarrow \alpha b \beta-\beta b \alpha=0
\end{aligned}
$$

for all $a, b, c \in M$ and $\alpha, \beta \in \Gamma$. Thus, the prime $M$-ring $\Gamma$ is commutative. Let $x, y \in M$ and $\gamma \in \Gamma$. Then,

$$
\begin{aligned}
\alpha(x \gamma y-y \gamma x) \beta & =\alpha x \gamma y \beta-\alpha y \gamma x \beta=\alpha x \gamma y \beta-\beta x \alpha y \gamma \\
& =\alpha x \gamma y \beta-\beta x \gamma y \alpha=\alpha x \gamma y \beta-\alpha x \gamma y \beta \\
& =0
\end{aligned}
$$

for all $\alpha, \beta \in \Gamma$, since $\Gamma$ is commutative $M$-ring. Therefore, we get $x \gamma y=y \gamma x$ for all $x, y \in M$ and $\gamma \in \Gamma$. Hence, $M$ is a commutative $\Gamma$-ring.

Theorem 2.2. Let $M$ be a prime weak Nobusawa $\Gamma$-ring. If a $\alpha b \beta c=a \beta b \alpha c$ for all $a, b, c \in M$ and $\alpha, \beta \in \Gamma$, then $(\Gamma, M)_{w N}$ is commutative.

Proof. Since $M$ is a weak Nobusawa $\Gamma$-ring, $M$ is a Nobusawa $\Gamma^{\prime}$-ring by Lemma 1.3. Then, $\Gamma^{\prime}$ is a prime Nobusawa $M$-ring by Lemma 1.5. Since $a \alpha b \beta c=a \beta b \alpha c$, we get

$$
a[\alpha, x, \beta] b\left[\alpha^{\prime}, x^{\prime}, \beta^{\prime}\right] c=a\left[\alpha^{\prime}, x^{\prime}, \beta^{\prime}\right] b[\alpha, x, \beta] c
$$

for all $a, b, c, x, x^{\prime} \in M$ and $\alpha, \beta, \alpha^{\prime}, \beta^{\prime} \in \Gamma$. Hence, by Lemma 2.1 we have $\left(\Gamma^{\prime}, M\right)_{N}$ is commutative that is, $a[\alpha b \beta] c=c[\alpha b \beta] a$ for all $a, b, c \in M$ and $\alpha, \beta \in \Gamma$. Let $[\gamma, x, \beta]$ and $\left[\gamma^{\prime}, x^{\prime}, \beta^{\prime}\right]$ in $\Gamma^{\prime}, a, b \in M$ and $\alpha \in \Gamma$. It suffices to show that the equation

$$
[\gamma, x, \beta](a \alpha b-b \alpha a)\left[\gamma^{\prime}, x^{\prime}, \beta^{\prime}\right]=\left[\gamma, x \beta(a \alpha b-b \alpha a) \gamma^{\prime} x^{\prime}, \beta^{\prime}\right]=0_{\Gamma^{\prime}}
$$

holds.

Let $u, v \in M$. Then we have,

$$
\begin{aligned}
u \gamma x \beta(a \alpha b-b \alpha a) \gamma^{\prime} x^{\prime} \beta^{\prime} v & =(u(\gamma x \beta) a \alpha b) \gamma^{\prime} x^{\prime} \beta^{\prime} v-u \gamma x \beta b \alpha a \gamma^{\prime} x^{\prime} \beta^{\prime} v \\
& =(b \gamma x \beta a) \alpha u \gamma^{\prime} x^{\prime} \beta^{\prime} v-u \gamma x \beta b \alpha a \gamma^{\prime} x^{\prime} \beta^{\prime} v \\
& =(a(\gamma x \beta) b \alpha u) \gamma^{\prime} x^{\prime} \beta^{\prime} v-u \gamma x \beta b \alpha a \gamma^{\prime} x^{\prime} \beta^{\prime} v \\
& =u \gamma x \beta b \alpha a \gamma^{\prime} x^{\prime} \beta^{\prime} v-u \gamma x \beta b \alpha a \gamma^{\prime} x^{\prime} \beta^{\prime} v \\
& =0
\end{aligned}
$$

for all $u, v \in M$. Therefore, $\left(\gamma, x \beta(a \alpha b-b \alpha a) \gamma^{\prime} x^{\prime}, \beta^{\prime}\right) \in \Lambda$. Hence, we get $a \alpha b=b \alpha a$ for all $a, b \in M$. This shows that $(\Gamma, M)_{w N}$ is commutative.

Lemma 2.3. Let $M$ be a prime Nobusawa $\Gamma$-ring and $U$ be a nonzero ideal of $M$. If $u \gamma v=0$ for all $u, v \in U$, then $\gamma=0$.

Proof. Since $U \Gamma M \gamma M \Gamma U=0$ by hypothesis, it can be shown that $\gamma=0$ since $U$ is a nonzero ideal of $M$ and $M$ is prime.

Theorem 2.4. Let $M$ be a $\Gamma$-ring in the sense of Nobusawa. Then, $M$ is a commutative $\Gamma$-ring if and only if $\Gamma$ is a commutative $M$-ring where $M$ and $\Gamma$ both nonzero.

Proof. We only prove that if $M$ is a commutative $\Gamma$-ring, then $\Gamma$ is a commutative $M$ ring. By Lemma 1.5, we have $\Gamma$ is an $M$-ring in the sense of Nobusawa. Thus, we only need to show the commutativity of the $M$-ring $\Gamma$. 
Suppose that $M$ is a commutative $\Gamma$-ring. Therefore, $[x, y]_{\alpha}=0$ for all $x, y \in M$ and $\alpha \in \Gamma$. Replacing $x$ by $x \beta z$ for $z \in M$ and $\beta \in \Gamma$, we get $x[\beta, \alpha]_{y} z=0$ for all $x, y, z \in M$ and $\alpha, \beta \in \Gamma$. Hence, $[\beta, \alpha]_{y}=0$ for all $y \in M$ and $\alpha, \beta \in \Gamma$ since $M$ is a Nobusawa $\Gamma$-ring. This implies that the $M$-ring $\Gamma$ is commutative.

Remark 2.5. From now on, in the light of the Lemma 1.5 and Theorem 2.4, the lemmas and the theorems proved for a $\Gamma$-ring $M$ are also valid for an $M$-ring $\Gamma$.

Lemma 2.6. Let $M$ be a $\Gamma$-ring in the sense of Barnes and $x$ be a nonzero element of $C_{\Gamma}(M)$. Then the equation,

$$
\operatorname{x\alpha y}[a \beta b, c]_{\gamma}=x \alpha y \delta\left(a \beta[b, c]_{\gamma}+[a, c]_{\gamma} \beta b\right)
$$

holds for all $a, b, c, y \in M$ and $\alpha, \beta, \gamma, \delta \in \Gamma$.

Proof. It suffices to show that

$$
x \alpha y \delta a \beta c \gamma b-x \alpha y \delta a \gamma c \beta b=0
$$

since

$$
[a \beta b, c]_{\gamma}=a \beta[b, c]_{\gamma}+[a, c]_{\gamma} \beta b+a[\beta, \gamma]_{c} b
$$

for all $a, b, c, y \in M$ and $\alpha, \beta, \gamma, \delta \in \Gamma$. Thus,

$$
\begin{aligned}
x \alpha y \delta a \beta c \gamma b & =y \alpha x \delta a \beta c \gamma b=y \alpha a \delta x \beta c \gamma b \\
& =y \alpha a \delta c \beta x \gamma b=y \alpha a \delta c \beta b \gamma x \\
& =y \alpha a \delta x \gamma c \beta b=y \alpha x \delta a \gamma c \beta b \\
& =x \alpha y \delta a \gamma c \beta b
\end{aligned}
$$

since $x \in C_{\Gamma}(M)$. Therefore, we get the desired result.

Corollary 2.7. Let $M$ be a prime $\Gamma$-ring in the sense of Barnes and $x$ be a nonzero element of $C_{\Gamma}(M)$. Then the equations,

$$
\begin{aligned}
& {[a \beta b, c]_{\gamma}=a \beta[b, c]_{\gamma}+[a, c]_{\gamma} \beta b} \\
& {[a, b \beta c]_{\gamma}=\beta b[a, c]_{\gamma}+[a, b]_{\gamma} \beta c}
\end{aligned}
$$

hold for all $a, b, c \in M$ and $\beta, \gamma \in \Gamma$.

Corollary 2.8. Let $M$ be a prime $\Gamma$-ring in the sense of Nobusawa. Then the center of $M$ is either zero or $M$.

Proof. Let $C_{\Gamma}(M) \neq 0$. Then, we have the equation

$$
[a \beta b, c]_{\gamma}=a \beta[b, c]_{\gamma}+[a, c]_{\gamma} \beta b
$$

by Corollary 2.7. This implies that $a \beta c \gamma b=a \gamma c \beta b$ for all $a, b, c \in M$ and $\beta, \gamma \in \Gamma$. Therefore, by Lemma 2.1 , the center of $M$ equals to $M$.

Lemma 2.9. If $R$ is a prime ring, then $R$ is a prime $R$-ring in the sense of Nobusawa.

Proof. It is obvious that the ternary multiplications can be defined as the multiplication of the ring $R$ and therefore one can easily see that $R$ is a Nobusawa $R$-ring. On the other hand, the primeness of the $R$-ring $R$ is clear since of $R$ is prime as a ring.

Lemma 2.10. Let $R$ be a prime ring. Then, $C_{R}(R)$ is contained in $Z(R)$.

Proof. Let $a \in C_{R}(R)$. Hence, $a x y-y x a=0$ for all $x, y \in R$. Replacing $y$ by $y z$ in the last equation yields,

$$
y(x a-a x) z=0
$$

for all $x, y, z \in R$. Thus, we have $x a-a x=0$ for all $x \in R$ since $R$ is an $R$-ring in the sense of Nobusawa by Lemma 2.9. This implies that $a \in Z(R)$. Therefore, $C_{R}(R)$ is contained in $Z(R)$. 
Theorem 2.11. Let $R$ be a prime ring with $C_{R}(R) \neq 0$. Then, $R$ is commutative.

Proof. Since $R$ is a nonzero prime ring, we have a commutative and prime $R$-ring $R$ by Lemma 2.9 and Corollary 2.8. Therefore, $[x, y]_{r}=0$ for all $x, y, r \in R$. By Lemma 2.10, we have a nonzero element $c$ in $Z(R)$. Replacing $y$ by $c$ in the last equation, we get $c[x, r]=0$ for all $x, r \in R$ since $c \in Z(R)$. Since $R$ is prime, $R$ is also commutative as a ring.

Remark 2.12. Let $R$ be a prime ring. According to the Theorem 2.11, it is unnecessary to investigate its commutativity conditions if it satisfies $C_{R}(R) \neq 0$.

Lemma 2.13. Let $M$ be a prime $\Gamma$-ring in the sense of Nobusawa, $U$ be a nonzero ideal of $M$ and $\Omega$ be a nonzero ideal of $\Gamma$.

(i) If $x \Omega y=0$ for any $x, y \in M$, then $x=0$ or $y=0$.

(ii) If $\gamma U \beta=0$ for any $\gamma, \beta \in \Gamma$, then $\gamma=0$ or $\beta=0$.

Proof. We only prove (i). The other case can be shown similarly.

Suppose that $x \Omega y=0$. Thus, $x \Omega M=0$ or $y=0$ since $\Omega$ is an ideal of $\Gamma$ and $M$ is prime. Let $x \Omega M=0$. Since $M$ is prime and $\Omega$ is nonzero, we get $x=0$.

Lemma 2.14. Let $M$ be a prime $\Gamma$-ring in the sense of Nobusawa, $U$ be a nonzero ideal of $M$ and $\Omega$ be a nonzero ideal of $\Gamma$. Then $M$ is commutative if one of the following conditions holds:

(i) $C_{U}(\Gamma) \neq 0$

(ii) $C_{\Gamma}(U) \neq 0$

(iii) $C_{\Omega}(M) \neq 0$

(iv) $C_{M}(\Omega) \neq 0$

Proof. Here we prove the theorem under the conditions (i) or (ii). The same proof could be carried out under the other conditions.

(i) Suppose that $\gamma$ is a nonzero element of $C_{U}(\Gamma)$. So we have $\gamma u \delta-\delta u \gamma=0$ for all $u \in U$ and $\delta \in \Gamma$. Then,

$$
\begin{aligned}
\gamma u[\delta, \beta]_{v} & =\gamma u \delta v \beta-\gamma u \beta v \delta \\
& =\delta u \gamma v \beta-\delta u \beta v \gamma \\
& =\delta u \beta v \gamma-\delta u \beta v \gamma \\
& =0
\end{aligned}
$$

for all $u, v \in U$ and $\delta, \beta \in \Gamma$. Hence, we get $[\delta, \beta]_{v}=0$ for all $v \in U$ and $\delta, \beta \in \Gamma$ by Lemma 2.13. Now let $y \in M$. Since $U$ is an ideal of $M$, we have

$$
\begin{aligned}
\gamma u[\delta, \beta]_{y} & =\gamma u \delta y \beta-\gamma u \beta y \delta=\beta u \delta y \gamma-\delta u \beta y \gamma \\
& =\delta u \beta y \gamma-\beta u \delta y \gamma=\beta u \delta y \gamma-\beta u \delta y \gamma \\
& =0
\end{aligned}
$$

for all $u \in U, y \in M$ and $\delta, \beta \in \Gamma$. Thus, we get $[\delta, \beta]_{y}=0$ for all $y \in M$ and $\delta, \beta \in \Gamma$ by Lemma 2.13. Therefore, we have the desired result by Theorem 2.4.

(ii) Suppose that $x$ is a nonzero element of $C_{\Gamma}(U)$. So we have $[x, u]_{\gamma}=0$ for all $u \in U$ and $\gamma \in \Gamma$. Therefore, the equation

$$
[u \gamma v, x]_{\beta}=u \gamma[v, x]_{\beta}+u[\gamma, \beta]_{x} v+[u, x]_{\beta} \gamma v
$$

reduces to $u[\gamma, \beta]_{x} v=0$ for all $u, v \in U$ and $\gamma, \beta \in \Gamma$. Hence, $[\gamma, \beta]_{x}=0$ for all $\gamma, \beta \in \Gamma$ by Lemma 2.3. Since $[x, u \beta y]_{\gamma}=0$ by hypothesis, we get $u \beta[x, y]_{\gamma}=0$ for all $u \in U, y \in M$ and $\gamma, \beta \in \Gamma$. Thus, $x \in C_{\Gamma}(M)$ since $M$ is a prime Nobusawa $\Gamma$-ring. Therefore, $M$ is $\Gamma$-commutative by Corollary 2.8 . 
Theorem 2.15. Let $M$ be a prime $\Gamma$-ring in the sense of Nobusawa, $U$ be a nonzero ideal of $M$ and $\Omega$ be a nonzero ideal of $\Gamma$. Then $M$ is commutative if one of the following conditions holds:

(i) $C_{U}(\Omega) \neq 0$

(ii) $C_{\Omega}(U) \neq 0$

Proof. Here we prove the theorem under the condition (i). The same proof could be carried out under the other condition.

Suppose that $\gamma$ is a nonzero element of $C_{U}(\Omega)$. So we have $[\gamma, \delta]_{u}=0$ for all $u \in U$ and $\delta \in \Omega$. Hence, the equation

$$
[\delta u \beta, \gamma]_{v}=\delta u[\beta, \gamma]_{v}+\delta[u, v]_{\gamma} \beta+[\delta, \gamma]_{v} u \beta
$$

reduces to $\delta[u, v]_{\gamma} \beta=0$ for all $u, v \in U$ and $\delta, \beta \in \Omega$. Therefore, $[u, v]_{\gamma}=0$ by Lemma 2.3. Then, we find

$$
\begin{aligned}
\gamma u[\alpha, \beta]_{v} & =\gamma u \alpha v \beta-\gamma u \beta v \alpha=\beta u \alpha v \gamma-\beta u \gamma v \alpha \\
& =\gamma v \beta u \alpha-\beta v \gamma u \alpha=\gamma v \beta u \alpha-\gamma v \beta u \alpha \\
& =0
\end{aligned}
$$

for all $u, v \in U, \alpha \in \Gamma$ and $\beta \in \Omega$. This implies that $[\alpha, \beta]_{v}=0$ by Lemma 1.5 and $\gamma \neq 0$. Therefore, we get that the nonzero ideal $\Omega$ of $\Gamma$ is a subset of $C_{U}(\Gamma)$. Hence, $M$ is $\Gamma$-commutative by Lemma 2.14 .

Corollary 2.16. Let $M$ be a prime $\Gamma$-ring in the sense of Nobusawa and $U$ be a nonzero ideal of $M$. If there exists a nonzero element $\gamma$ of $\Gamma$ such that $[U, M]_{\gamma}=0$, then $M$ is Г-commutative.

Proof. Suppose that there exists a nonzero element $\gamma$ of $\Gamma$ such that $[U, M]_{\gamma}=0$. Therefore, we have $y[\gamma, \beta]_{u} z=0$ for all $u \in U, y, z \in M$ and $\beta \in \Gamma$ since $[u, y \beta z]_{\gamma}=0$ by hypothesis. Thus, $[\gamma, \beta]_{u}=0$ since $M$ is a Nobusawa $\Gamma$-ring. This implies that $C_{U}(\Gamma) \neq 0$. Hence, $M$ is $\Gamma$-commutative by Lemma 2.14 .

Corollary 2.17. Let $M$ be a prime $\Gamma$-ring in the sense of Nobusawa and $U$ be a nonzero ideal of $M$. If there exists a nonzero element $\gamma$ of $\Gamma$ such that $[u, v]_{\gamma}=0$ for all $u, v \in U$, then $M$ is $\Gamma$-commutative.

Proof. By hypothesis, we have $y[\gamma, \beta]_{x} z=0$ since $[x, y \beta z]_{\gamma}=0$ for all $x, y, z \in U$ and $\beta \in \Gamma$. This implies that $[\gamma, \beta]_{x}=0$ for all $x \in U$ and $\beta \in \Gamma$ by Lemma 2.3. Therefore, $C_{U}(\Gamma) \neq 0$. Hence, $M$ is $\Gamma$-commutative by Lemma 2.14 .

Acknowledgment. The authors would like to thank the referees for the careful and detailed reading of the manuscript and valuable suggestions and comments.

\section{References}

[1] W.E. Barnes, On the Г-rings of Nobusawa, Pacific J. Math. 18 (3), 411-422, 1966.

[2] W.E. Coppage and J. Luh, Radicals of gamma rings, J. Math. Soc. Japan, 23 (1), 40-52, 1971.

[3] K.K. Dey and A.C. Paul, Free Actions on Semiprime Gamma Rings, Gen. Math. Notes, 5 (1), 7-14, 2011.

[4] Md.F. Hoque and A.C. Paul, On Centralizers of Semiprime Gamma Rings, Int. Math. Forum, 6 (13), 627-638, 2011.

[5] S. Kyuno, On the radicals of gamma rings, Osaka J. Math. 12, 639-645, 1975.

[6] S. Kyuno, Gamma Rings, Hadronic Press, 1991.

[7] N. Nobusawa, On a generalization of the ring theory, Osaka J. Math. 1, 81-89, 1964. 